\title{
Property-Tuneable Microgels Fabricated by Using Flow-Focusing Microfluidic Geometry for Bioactive Agent Delivery
}

\author{
Wing-Fu Lai ${ }^{1,2, *(1)}$ and Wing-Tak Wong ${ }^{1}$ \\ 1 Department of Applied Biology and Chemical Technology, Hong Kong Polytechnic University, \\ Hong Kong, China; w.t.wong@polyu.edu.hk \\ 2 Ciechanover Institute of Precision and Regenerative Medicine, The Chinese University of Hong Kong (Shenzhen), \\ Shenzhen 518172, China \\ * Correspondence: rori0610@graduate.hku.hk
}

Citation: Lai, W.-F.; Wong, W.-T.

Property-Tuneable Microgels

Fabricated by Using Flow-Focusing Microfluidic Geometry for Bioactive Agent Delivery. Pharmaceutics 2021, 13, 787. https://doi.org/10.3390/ pharmaceutics13060787

Academic Editors: Susana C.M. Fernandes and Garbine Aguirre

Received: 15 April 2021

Accepted: 20 May 2021

Published: 25 May 2021

Publisher's Note: MDPI stays neutral with regard to jurisdictional claims in published maps and institutional affiliations.

Copyright: () 2021 by the authors. Licensee MDPI, Basel, Switzerland. This article is an open access article distributed under the terms and conditions of the Creative Commons Attribution (CC BY) license (https:// creativecommons.org/licenses/by/ $4.0 /)$.

\begin{abstract}
Gelatine methacryloyl (GM) shows high biocompatibility and is extensively used in tissue engineering; however, few works have explored the use of GM in bioactive agent delivery. This study adopts a microfluidic approach involving the use of flow-focusing microfluidic geometry for microgel fabrication. This approach generates highly monodisperse microgels whose size can be tuned by altering various fabrication conditions (including the concentration of the gel-forming solution and the flow rates of different phases). By using tetracycline hydrochloride as a model agent, the fabricated microgels enable prolonged agent release, with the encapsulation efficiency being around $30-40 \%$ depending on the concentration of the gel-forming solution. Along with their negligible cytotoxicity, our microgels show the potential to serve as carriers of bioactive agents for food and pharmaceutical applications.
\end{abstract}

Keywords: microgels; microfluidics; flow-focusing geometry; nutraceuticals; controlled release

\section{Introduction}

Gelatine is a hydrolysis product of collagen [1-3], but compared to collagen, it shows less antigenicity and higher aqueous solubility [4]. While a gelatine solution can undergo gelation to form physical hydrogels upon a decrease in temperature, few derivatives of gelatine are capable of forming chemical hydrogels [5]. A good example is gelatine methacryloyl (GM), which is a derivative generated upon conjugation of methacrylate groups to amine-containing groups of gelatine. One favourable property of GM is its high tunability in properties, which can be manipulated simply by changing various synthetic and processing parameters [4]. In addition, the surface of GM supports cell adherence and growth [6]. This, along with the possibility of GM to undergo polymerisation in mild conditions, enables GM to be widely adopted as scaffolds for cell encapsulation [7-9]. In fact, the properties of GM as mentioned above are favourable not only for tissue engineering but also for bioactive agent delivery. Along with its polymerisability upon UV irradiation, GM can potentially be used to generate microgels via emulsion polymerisation as carriers of bioactive agents.

Emulsion polymerisation is one of the commonly used approaches to generate microgels. During the process, high shear energy is applied to mix a gel-forming solution with an immiscible continuous phase to obtain emulsion particles for subsequent polymerisation reactions [10-12]. This method enables quick preparation of a large number of emulsion particles; however, the polydispersity of the generated microgels is high. Fragile bioactive agents (e.g., peptides and proteins) may also be damaged by high shear energy during the process of agent loading, which is generally performed concomitantly with the process of emulsion polymerisation. To address these problems, this study incorporates a microfluidic approach into the conventional process of emulsion polymerization to generate microgels for bioactive agent delivery. During microgel fabrication, an aqueous gel-forming solution 
flowing in one channel is subjected to the shear stress generated by the continuous oil phase flowing in another channel to produce microdroplets for subsequent photopolymerisation. By using flow-focusing microfluidic geometry, along with the manipulation of various fabrication conditions (including the concentration of the gel-forming solution and the flow rates of different phases), we have successfully produced microgels with different sizes and degrees of swelling for delivery of bioactive agents.

\section{Materials and Methods}

\subsection{Materials}

4-dimethylaminopyridine (DMAP), gelatine, and various other chemicals were purchased from Sigma-Aldrich (St. Louis, MO, USA). SU-8 2000 was purchased from MicroChem (Newton, MA, USA). Dulbecco's Modified Eagle's Medium (DMEM; Gibco, Grand Island, NE, USA), penicillin G-streptomycin sulphate (Life Technologies Corporation, Chicago, IL USA), and foetal bovine serum (FBS; Hangzhou Sijiqing Biological Engineering Materials Co., Ltd., Hangzhou, China) were used as the cell culture medium. Trypsin-EDTA (0.25\% trypsin-EDTA) was obtained from Invitrogen (Carlsbad, CA, USA).

\subsection{Synthesis of $G M$}

$7 \mathrm{~g}$ of gelatine and $0.35 \mathrm{~g}$ of DMAP were dissolved in $60 \mathrm{~mL}$ of dimethyl sulfoxide at $50{ }^{\circ} \mathrm{C}$. Then, $3 \mathrm{~mL}$ of glycidyl methacrylate was added dropwise under constant stirring at $50{ }^{\circ} \mathrm{C}$ in an inert nitrogen atmosphere. After $48 \mathrm{~h}$ of reaction, the mixture was dialysed against deionized (DI) water for 3 days before lyophilisation to obtain GM.

\subsection{Structural Characterisation}

GM was solubilized in deuterium oxide $\left(\mathrm{D}_{2} \mathrm{O}\right)$. Proton nuclear magnetic resonance $\left({ }^{1} \mathrm{H}\right.$ NMR) spectra were recorded using an NMR spectrometer $(500 \mathrm{MHz}$; Bruker Corporation, Rheinstetten, Germany). The structures of gelatine, GM and MM were also characterized by using Fourier-transform infrared (FT-IR) spectroscopy (Nicolet5700; Thermo Nicolet Company, Waltham, MA, USA) at ambient conditions. Spectra were reported as an average of 16 scans.

\subsection{Generation of Microgels by Using a Microfluidic Flow-Focusing Device}

A silicon wafer was spin-coated with SU-8 2000 and baked at $95^{\circ} \mathrm{C}$. A photomask with patterns for microfluidic channels was placed on top of the wafer, followed by UV irradiation to crosslink the patterned area. The crosslinked photoresist was further solidified upon baking at $95^{\circ} \mathrm{C}$. After that, the wafer was put into the SU-8 developer, and was rinsed with isopropanol. A silicone elastomer base and a curing agent were mixed in a 10:1 ratio. The mixture was poured onto the master. Upon curing at $80^{\circ} \mathrm{C}$ for $3 \mathrm{~h}$, a polydimethylsiloxane (PDMS) elastomer with engraved microchannels was detached from the master. A hole punch (diameter $=0.5 \mathrm{~mm}$ ) was adopted to produce the fluid inlet and outlet. A glass slide was irreversibly bonded to the PDMS elastomer upon surface treatment with oxygen plasma for 3 minutes to obtain a microfluidic device.

During microgel fabrication, plastic tubing (inner diameter $=0.3 \mathrm{~mm}$, outer diameter $=0.76 \mathrm{~mm}$ ) was used to connect the inlet and outlet of the device. The aqueous phase was prepared by dissolving GM (5 or 8\% $(w / v))$ and 2-hydroxy-4'-(2-hydroxyethoxy)2-methylpropiophenone $(0.2 \%(w / v))$ in phosphate-buffered saline (PBS, pH 7.4)); whereas the oil phase was prepared by mixing Span $80(20 \%(v / v))$ with mineral oil (Sigma Aldrich, St. Louis, MO, USA). Different phases were injected into the device with controlled flow rates by using syringe pumps (PHD 2000; Harvard Apparatus, Holliston, MA, USA). Microgels were generated upon UV irradiation of the droplets generated in the device. They were retrieved by centrifugation, followed by washing with PBS five times. Microgels were designated as MM, with those fabricated from $5 \%(w / v), 6.5 \%(w / v)$ and $8 \%(w / v)$ GM solutions being designated as MM50, MM65 and MM80, respectively. 


\subsection{Determination of the Swelling Behaviour}

Microgels were immersed in PBS. Changes in the diameter of the microgels were recorded under an inverted optical microscope (Eclipse TE2000-U; Nikon, Tokyo, Japan) at regular time intervals and were analysed by using Image J software.

\subsection{Thermogravimetric Analysis (TGA)}

TGA of gelatine, GM and MM was performed using a Q50 TGA analyser (TA Instruments, New Castle, DE, USA) equipped with platinum pans. Analysis was performed in an inert nitrogen atmosphere from $40{ }^{\circ} \mathrm{C}$ to $740{ }^{\circ} \mathrm{C}$. The heating rate was set as $10^{\circ} \mathrm{C} \mathrm{min}{ }^{-1}$.

\subsection{Evaluation of Cytotoxicity}

3T3 mouse fibroblasts and HEK293 cells were cultured as previously described [13]. The cells were seeded in a 96-well plate at a density of 5,000 cells per well. The plates were incubated at $37{ }^{\circ} \mathrm{C}$ under a humidified atmosphere of $5 \% \mathrm{CO}_{2}$ for $24 \mathrm{~h}$. Meanwhile, lyophilised MM80 was ground in DMEM using mortar and pestle to obtain a suspension with the desired concentration. The cell culture medium was replaced with $100 \mu \mathrm{L}$ of the suspension. After $5 \mathrm{~h}$ incubation at $37^{\circ} \mathrm{C}$, the suspension in each well was replaced with the fresh cell culture medium. The CellTiter 96 Aqueous Non-radioactive Cell Proliferation Assay (MTS assay; Promega Corp., Madison, WI, USA) was performed according to the manufacturer's instructions, either immediately or after $24 \mathrm{~h}$ of post-treatment incubation to determine the cell viability (\%) in each well.

\subsection{Determination of the Encapsulation Efficiency}

Tetracycline hydrochloride was adopted as a model agent. To form agent-loaded microgels, MM50, MM65 and MM80 were prepared as usual but tetracycline hydrochloride was added to the aqueous phase at a concentration of $0.6 \%(w / v)$ prior to microgel fabrication. The concentration of unloaded tetracycline hydrochloride was determined using a ultraviolet-visible (UV-Vis) spectrophotometer (Cary 300; Varian, Palo Alto, CA, USA) at $\lambda_{\max }$ of $360 \mathrm{~nm}$. The encapsulation efficiency (EE) was calculated using the following equation:

$$
E E(\%)=\frac{m_{l}}{m_{t}} \times 100 \%
$$

where $m_{l}$ is the mass of tetracycline hydrochloride encapsulated successfully by the microgels, and $m_{t}$ is the total mass of tetracycline hydrochloride added during the encapsulation process.

\subsection{Evaluation of the Kinetics of Agent Release}

The release sustainability of the microgels was evaluated based on a previously reported protocol [14]. In brief, $1 \mathrm{~g}$ of lyophilised, agent-loaded microgels was placed in $10 \mathrm{~mL}$ of PBS (pH 7.4) and incubated at $37^{\circ} \mathrm{C}$. At regular time intervals, $1 \mathrm{~mL}$ of the release medium was withdrawn and replenished by the same amount of PBS. The amount of tetracycline hydrochloride released from the microgels was analysed using a UV-Vis spectrophotometer (Cary 300; Varian, Palo Alto, CA, USA) at $\lambda_{\max }$ of $360 \mathrm{~nm}$. The percentage of cumulative agent release was calculated using the following equation:

$$
\text { Cumulative release }(\%)=\frac{\sum_{\mathrm{t}=0}^{\mathrm{t}} m_{t}}{m_{\infty}} \times 100 \%
$$

where $m_{t}$ is the mass of tetracycline hydrochloride released from the microgels at time $t$, and $m_{\infty}$ is the total mass of tetracycline hydrochloride loaded into the microgels. The release curves were fitted into different kinetic models (including the zero-order model, the first-order model, the Higuchi model and the Korsmeyer-Peppas model) to analyse the mechanism of agent release. 


\subsection{Statistical Analysis}

All data were presented as the means \pm standard deviations of triplicate experiments. Student's $t$-test was performed to assess the statistical significance. Differences with a $p$-value $<0.05$ were considered to be statistically significant.

\section{Results}

\subsection{Microgel Generation and Structural Characterisation}

GM is a derivative of gelatine. The successful incorporation of methacryloyl substituent groups into gelatine was confirmed by ${ }^{1} \mathrm{H}-\mathrm{NMR}$, in which the spectrum of GM shows peaks at 5.8 and $6.2 \mathrm{ppm}$ (Figure 1A). These peaks were assigned to the acrylic protons of the grafted methacryloyl group. In addition, a peak was found at $1.9 \mathrm{ppm}$. This peak was contributed by the methyl proton signal of the grafted methacryloyl group. GM was adopted for the generation of microgels in a PDMS-based microfluidic device (Figure 1B). During microgel formation, a pre-gel solution containing GM and 2-hydroxy-4'(2-hydroxyethoxy)-2-methylpropiophenone was used as the aqueous phase while mineral oil containing Span 80 was used as the oil phase (Figure 1C).

(A)

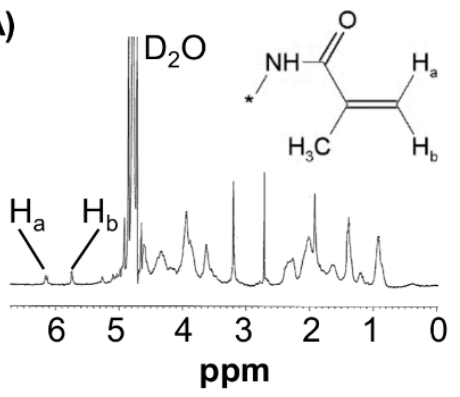

(B)

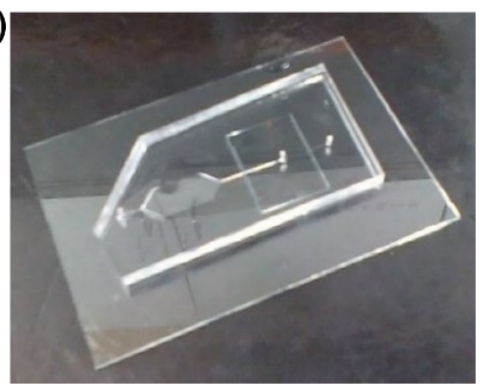

(C)

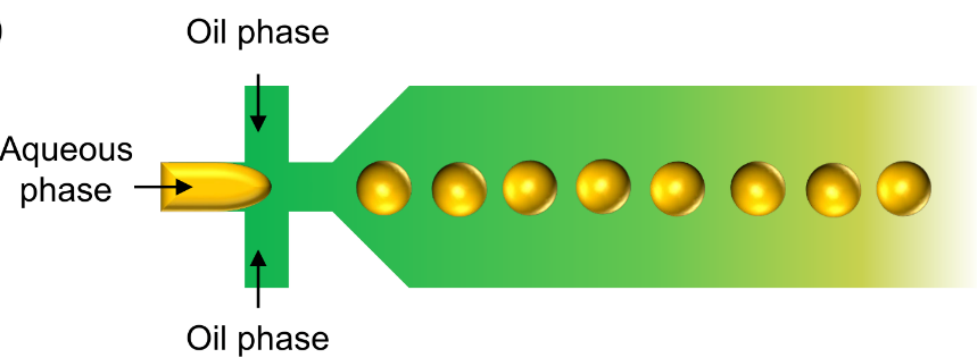

Figure 1. (A) ${ }^{1} \mathrm{H}-\mathrm{NMR}$ spectrum of GM. (B) A photo of the microfluidic device fabricated for microgel generation. (C) A schematic diagram depicting flow-focusing microfluidic geometry.

In the microfluidic device, the aqueous phase flowed through one channel and was intersected by the oil flow. A high concentration of Span 80 was adopted in this study to increase the viscosity of the oil phase so that adequate shear stress could be generated to produce aqueous droplets. In addition, the presence of a high concentration of Span 80 helped stabilise the aqueous droplets and prevent coalescence for the subsequent formation of monodisperse microgels for bioactive agent delivery (Figure 2A). The structure of the fabricated microgels, along with those of gelatine and GM, were characterised by using FT-IR (Figure 2B). In the spectrum of gelatine, a peak was found at around $1617 \mathrm{~cm}^{-1}$. This amide I band was attributed to $\mathrm{C}=\mathrm{O}$ stretching vibrations of the amide group. In addition, a signal was detected at $1540 \mathrm{~cm}^{-1}$ and was assigned to $\mathrm{N}-\mathrm{H}$ bending vibrations and $\mathrm{C}-\mathrm{H}$ stretching vibrations. Similar peaks were also found in the spectrum of GM, which displayed characteristic bands at around $1631 \mathrm{~cm}^{-1}$ (amide I), $1556 \mathrm{~cm}^{-1}$ (amide II). In the spectrum of GM, a signal was detected at around $1720 \mathrm{~cm}^{-1}$. This signal was attributed to the carbonyl signal from the methacrylate group $(\mathrm{C}=\mathrm{O}$ stretching) and was also found in the spectrum of MM. The methacrylate group in GM and MM was expected to be the functional group that enabled photo-crosslinking during microgel formation. 

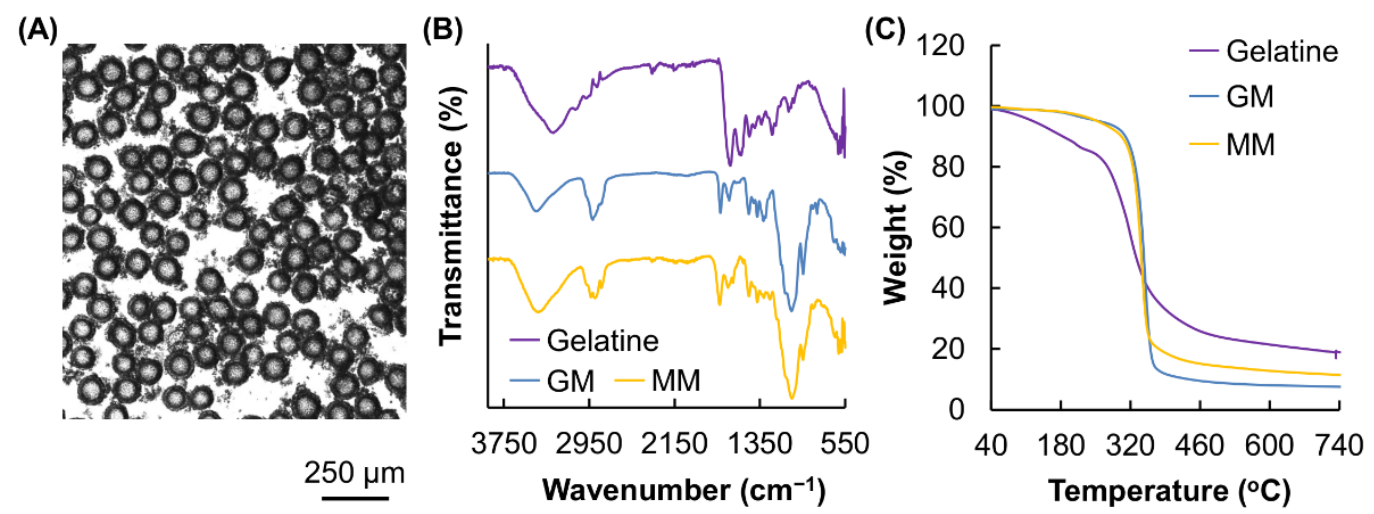

Figure 2. (A) A microscopic image of MM. (B) FTIR spectra of gelatine, GM and MM. (C) TGA curves of gelatine, GM and MM.

\subsection{Thermal Properties and Size Control}

The thermal properties of gelatine, as well as those of GM and MM, were characterised by using TGA (Figure 2C). The curve of gelatine exhibited two stages of weight loss. The first stage occurred below $220{ }^{\circ} \mathrm{C}$, accounting for a weight loss of around $13.5 \%$. This weight loss step was attributed to the loss of adsorbed moisture. The second stage occurred at the temperature range of $258-485{ }^{\circ} \mathrm{C}$, leading to a weight loss of around $60 \%$. In the curves of GM and MM, the percentage of weight loss in the first stage of the decomposition process from 50 to $220^{\circ} \mathrm{C}$ was significantly reduced as compared to that of gelatine, suggesting an increase in the hydrophobicity of gelatine after structural modification. This is consistent with the observation made previously by Rajitha and colleagues [15], who modified gelatine with diethylenetriamine (DETA) and found that an increase in the hydrophobicity of gelatine leads to a decrease in the percentage of weight loss in the stage of moisture evaporation.

The use of a flow-focusing microfluidic device for microgel fabrication in this study enabled the generation of microgels with controlled size. The size of the microgels was manipulated by varying the ratio of the flow rates of the aqueous and oil phases $\left(Q_{a q} / Q_{0}\right)$, and hence the magnitude of shear stress applied to the aqueous flow (Figure 3). Our results showed that the $Q_{a q} / Q_{o}$ values are positively related to the microgel diameter (Figure 4 ) and are negatively related to the surface-area-to-volume ratio $(S A: V)$ of the microgels formed (Figure 5). The concentration of GM in the aqueous phase also had a positive relationship with the size of the generated microgels. This was attributed to the fact that an increase in the GM concentration led to an increase in viscosity, leading to the formation of larger droplets. The size of the microgels further increased upon immersion in PBS, leading to a reduction in the $S A: V$ values. 


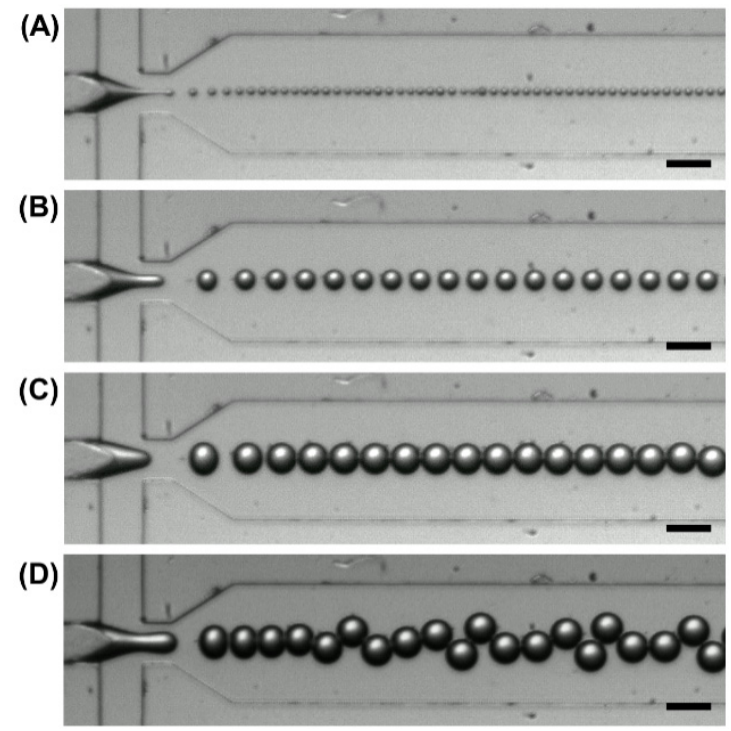

Figure 3. The size of MM fabricated at different $Q_{a q} / Q_{o}$ values: (A) 0.05; (B) 0.1 ; (C) 0.2; (D) 0.3. The concentration of GM in the aqueous phase is $8 \%(w / v)$. Scale bar is $100 \mu \mathrm{m}$.

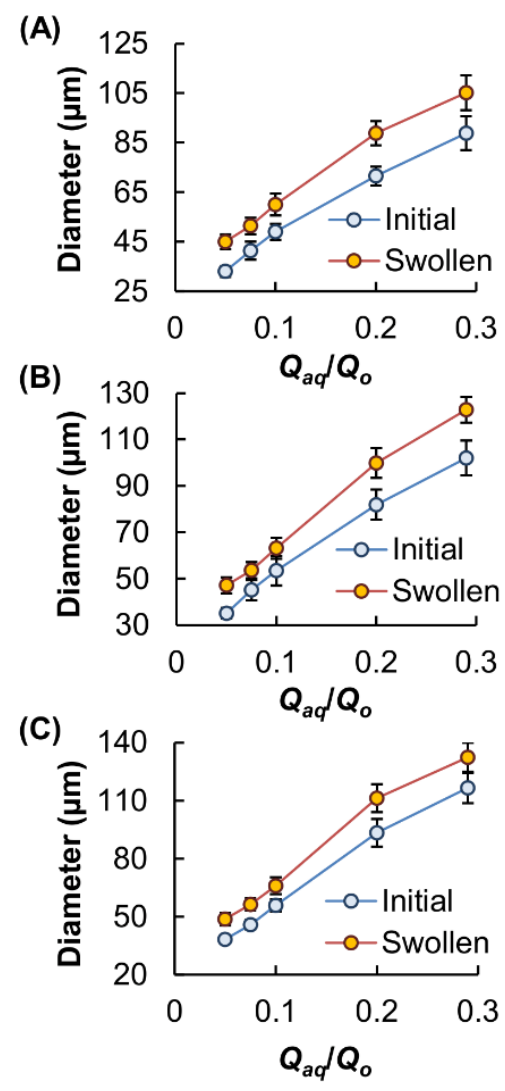

Figure 4. Changes in the average diameter of MM [(A) MM50, (B) MM65 and (C) MM80] upon changes in the $Q_{a q} / Q_{o}$ value. The diameter was measured immediately after microgel fabrication and after swelling in PBS for $24 \mathrm{~h}$. 

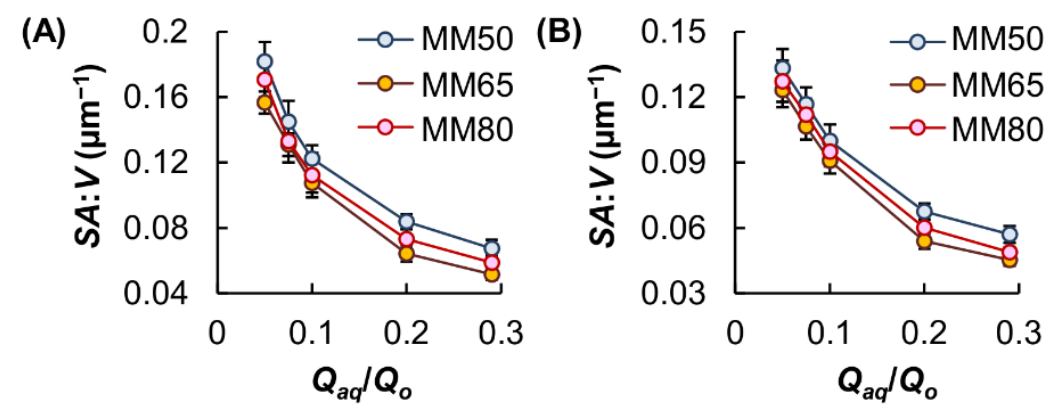

Figure 5. Changes in the $S A: V$ value of MM upon changes in the $Q_{a q} / Q_{o}$ value. The $S A: V$ value was measured either (A) immediately after microgel fabrication or (B) after swelling in PBS for $24 \mathrm{~h}$.

\subsection{Performance in Bioactive Agent Delivery}

In order to be used as carriers, the microgels have to possess a high safety profile [16-19]. The toxicity of the microgels was examined in vitro by using the MTS assay. No significant loss of cell viability was observed after $5 \mathrm{~h}$ treatment with the microgels. This indicated that the microgels have negligible acute cytotoxicity (Figure 6). To determine the chronic cytotoxicity of the microgels, the viability of the treated cells was further studied after $24 \mathrm{~h}$ post-treatment incubation. No detectable cytotoxicity was observed in all concentrations tested. This illustrated the negligible toxicity of the microgels for biological applications.

(A)

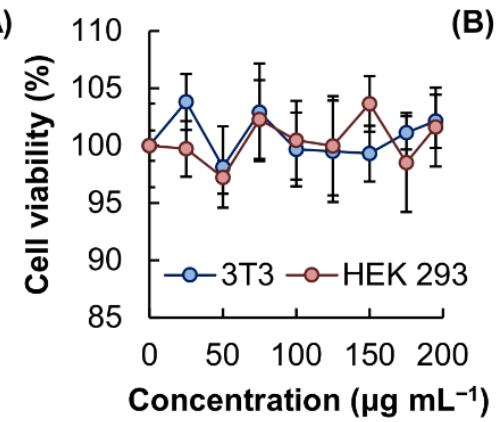

(B)

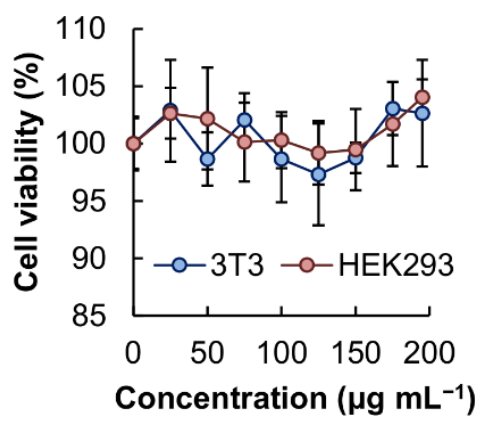

Figure 6. Viability of 3 T3 fibroblasts and HEK 293 cells after $5 \mathrm{~h}$ treatment with MM, (A) before and (B) after $24 \mathrm{~h}$ post-treatment incubation.

To evaluate the performance of the microgels in bioactive agent delivery, tetracycline hydrochloride was adopted as a model agent. Depending on the concentration of GM in the microgels, the EE was estimated to be around 30-40\% (Figure 7A). Among the microgels tested, MM80 displayed the highest sustainability of agent release (Figure 7B). This was partially attributed to the fact that, under the same $Q_{a q} / Q_{o}$ value, microgels generated from a pre-gel solution containing a lower concentration of GM have a smaller size. Because a reduction in the particle size may cause a decrease in the diffusion length and an increase in the $S A: V$ value [20-23], this favours the diffusion of the loaded molecules from the microgels to the surrounding medium and hence reduces the sustainability of agent release [24].

Upon fitting the curves of agent release into various kinetic models (including the zero-order model, the first-order model, the Higuchi model and the Korsmeyer-Peppas model) and based on the calculated regression coefficient $\left(r^{2}\right)$ values (Table 1$)$, the release profiles of the agent-loaded microgels were found to fit the Higuchi model the most. This suggested that the process of agent release involves the penetration of the release medium into the hydrogel matrix. In addition, the release exponents $(n)$, as calculated by using the Korsmeyer-Peppas' equation, were 0.611, 0.516 and 0.461 for MM80, MM65 and MM50, respectively. This indicated that the release of tetracycline hydrochloride from the 
microgels is controlled by multiple processes (including polymer relaxation and agent diffusion), with anomalous non-Fickian transport being the major release mechanism.

(A)

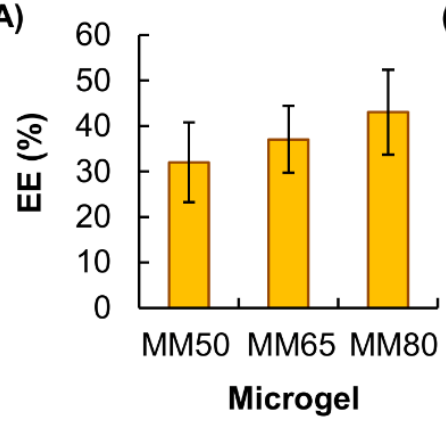

(B)

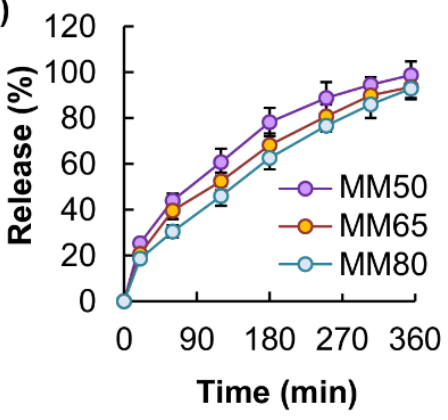

Figure 7. (A) The EE of MM50, MM65 and MM80. (B) Release profiles of tetracycline hydrochloride from MM50, MM65 and MM80.

Table 1. Correlation coefficients $\left(r^{2}\right)$ and release exponent $(n)$ of different models for the kinetic data of agent release.

\begin{tabular}{ccccc}
\hline \multirow{2}{*}{ Model } & & \multicolumn{3}{c}{ Microgel } \\
\cline { 3 - 5 } & & MM80 & MM65 & MM50 \\
\hline Zero-order model & $r^{2}$ & 0.912 & 0.832 & 0.758 \\
First-order model & $r^{2}$ & 0.985 & 0.980 & 0.984 \\
Higuchi model & $r^{2}$ & 0.986 & 0.998 & 0.994 \\
Korsmeyer-Peppas model & $r^{2}$ & 0.998 & 0.998 & 0.996 \\
& $n$ & 0.611 & 0.516 & 0.461 \\
\hline
\end{tabular}

\section{Conclusions}

Gelatine has a track record of applications in food, cosmetic and pharmaceutical industries [25-27]. To enhance the capacity of gelatine to form hydrogels, various derivatives have been generated over the years. One of them is GM, whose hydrogels have been widely adopted as scaffolds in tissue engineering [28-30]. In this study, we explored the potential use of GM in bioactive agent delivery, and adopted a microfluidic method to form monodisperse microgels. The size, as well as the $S A: V$ value of the microgels, could be tuned not only by changing the $Q_{a q} / Q_{o}$ value but also by manipulating the concentration of GM in the gel-forming solution. Such high tunability in properties, along with the high biocompatibility of GM, make our microgels worth being further exploited for food and pharmaceutical applications.

Author Contributions: Conceptualization, W.-F.L. and W.-T.W.; formal analysis, W.-F.L.; supervision, W.-F.L.; project administration, W.-F.L.; funding acquisition, W.-F.L. and W.-T.W. Both authors have read and agreed to the published version of the manuscript.

Funding: This research was funded by the Chinese University of Hong Kong, Shenzhen (PF01001421, UDF01001421 and UF02001421) and the Research Grants Council of the Government of Hong Kong Special Administrative Region (C5012-15E).

Institutional Review Board Statement: Not applicable.

Informed Consent Statement: Not applicable.

Data Availability Statement: Data are contained within the article.

Conflicts of Interest: The authors declare no conflict of interest. The funders had no role in the design of the study; in the collection, analyses, or interpretation of data; in the writing of the manuscript, or in the decision to publish the results. 


\section{References}

1. Madkhali, O.; Mekhail, G.; Wettig, S.D. Modified gelatin nanoparticles for gene delivery. Int. J. Pharm. 2019, 554, 224-234. [CrossRef] [PubMed]

2. Echave, M.C.; Saenz del Burgo, L.; Pedraz, J.L.; Orive, G. Gelatin as biomaterial for tissue engineering. Curr. Pharm. Des. 2017, 23, 3567-3584. [CrossRef] [PubMed]

3. Echave, M.C.; Hernaez-Moya, R.; Iturriaga, L.; Pedraz, J.L.; Lakshminarayanan, R.; Dolatshahi-Pirouz, A.; Taebnia, N.; Orive, G. Recent advances in gelatin-based therapeutics. Expert Opin. Biol. Ther. 2019, 19, 773-779. [CrossRef] [PubMed]

4. Yue, K.; Trujillo-de Santiago, G.; Alvarez, M.M.; Tamayol, A.; Annabi, N.; Khademhosseini, A. Synthesis, properties, and biomedical applications of gelatin methacryloyl (GelMA) hydrogels. Biomaterials 2015, 73, 254-271. [CrossRef] [PubMed]

5. Occhetta, P.; Visone, R.; Russo, L.; Cipolla, L.; Moretti, M.; Rasponi, M. VA-086 methacrylate gelatine photopolymerizable hydrogels: A parametric study for highly biocompatible 3D cell embedding. J. Biomed. Mater. Res. A 2015, 103, 2109-2117. [CrossRef]

6. Benton, J.A.; DeForest, C.A.; Vivekanandan, V.; Anseth, K.S. Photocrosslinking of gelatin macromers to synthesize porous hydrogels that promote valvular interstitial cell function. Tissue Eng. Pt. A 2009, 15, 3221-3230. [CrossRef]

7. Mehrali, M.; Thakur, A.; Kadumudi, F.B.; Pierchala, M.K.; Cordova, J.A.V.; Shahbazi, M.A.; Mehrali, M.; Pennisi, C.P.; Orive, G.; Gaharwar, A.K.; et al. Pectin methacrylate (PEMA) and gelatin-based hydrogels for cell delivery: Converting waste materials into biomaterials. ACS Appl. Mater. Int. 2019, 11, 12283-12297. [CrossRef]

8. Puckert, C.; Tomaskovic-Crook, E.; Gambhir, S.; Wallace, G.G.; Crook, J.M.; Higgins, M.J. Molecular interactions and forces of adhesion between single human neural stem cells and gelatin methacrylate hydrogels of varying stiffness. Acta Biomater. 2020, 106, 156-169. [CrossRef]

9. Ciortan, L.; Macarie, R.D.; Cecoltan, S.; Vadana, M.; Tucureanu, M.M.; Mihaila, A.C.; Droc, I.; Butoi, E.; Manduteanu, I. Chronic high glucose concentration induces inflammatory and remodeling changes in valvular endothelial cells and valvular interstitial cells in a gelatin methacrylate 3D model of the human aortic valve. Polymers 2020, 12, 2786. [CrossRef]

10. Dupin, D.; Fujii, S.; Armes, S.P.; Reeve, P.; Baxter, S.M. Efficient synthesis of sterically stabilized pH-responsive microgels of controllable particle diameter by emulsion polymerization. Langmuir 2006, 22, 3381-3387. [CrossRef]

11. Antonietti, M.; Bremser, W.; Mueschenborn, D.; Rosenauer, C.; Schupp, B.; Schmidt, M. Synthesis and size control of polystyrene latices via polymerization in microemulsion. Macromolecules 1991, 24, 6636-6643. [CrossRef]

12. Antonietti, M.; Bremser, W.; Schmidt, M. Microgels: Model polymers for the crosslinked state. Macromolecules 1990, 23 , 3796-3805. [CrossRef]

13. Lai, W.F.; Susha, A.S.; Rogach, A.L.; Wang, G.A.; Huang, M.J.; Hu, W.J.; Wong, W.T. Electrospray-mediated preparation of compositionally homogeneous core-shell hydrogel microspheres for sustained drug release. RSC Adv. 2017, 7, 44482-44491. [CrossRef]

14. Lai, W.F.; Huang, E.; Wong, W.T. A gel-forming clusteroluminogenic polymer with tunable emission behavior as a sustainedrelease carrier enabling real-time tracking during bioactive agent delivery. Appl. Mater. Today 2020, 21, 100876. [CrossRef]

15. Rajitha, K.; Mohana, K.N.S.; Mohanan, A.; Madhusudhana, A.M. Evaluation of anti-corrosion performance of modified gelatingraphene oxide nanocomposite dispersed in epoxy coating on mild steel in saline media. Colloid Surf. A 2020, 587, 124341. [CrossRef]

16. Lai, W.F.; Rogach, A.L.; Wong, W.T. One-pot synthesis of an emulsion-templated hydrogel-microsphere composite with tunable properties. Compos. Part A-Appl. Sci. Manuf. 2018, 113, 318-329. [CrossRef]

17. Lai, W.F.; Tang, R.; Wong, W.T. Ionically crosslinked complex gels loaded with oleic acid-containing vesicles for transdermal drug delivery. Pharmaceutics 2020, 12, 725. [CrossRef] [PubMed]

18. Lai, W.F.; Wong, E.; Wong, W.T. Multilayered composite-coated ionically crosslinked food-grade hydrogel beads generated from algal alginate for controlled and sustained release of bioactive compounds. RSC Adv. 2020, 10, 44522-44532. [CrossRef]

19. Lai, W.F.; Wong, W.T. Design of polymeric gene carriers for effective intracellular delivery. Trends Biotechnol. 2018, 36, 713-728. [CrossRef]

20. Webber, M.J.; Pashuck, E.T. (Macro)molecular self-assembly for hydrogel drug delivery. Adv. Drug. Deliv. Rev. 2021, 172, 275-295. [CrossRef]

21. Trombino, S.; Servidio, C.; Curcio, F.; Cassano, R. Strategies for hyaluronic acid-based hydrogel design in drug delivery. Pharmaceutics 2019, 11, 407. [CrossRef] [PubMed]

22. Patel, G.C.; Dalwadi, C.A. Recent patents on stimuli responsive hydrogel drug delivery system. Recent Pat. Drug. Deliv. Formul. 2013, 7, 206-215. [CrossRef] [PubMed]

23. Hamidi, M.; Azadi, A.; Rafiei, P. Hydrogel nanoparticles in drug delivery. Adv. Drug. Deliv. Rev. 2008, 60, 1638-1649. [CrossRef]

24. Lai, W.F.; Shum, H.C. A stimuli-responsive nanoparticulate system using poly(ethylenimine)-graft-polysorbate for controlled protein release. Nanoscale 2016, 8, 517-528. [CrossRef] [PubMed]

25. Calixto, S.; Ganzherli, N.; Gulyaev, S.; Figueroa-Gerstenmaier, S. Gelatin as a photosensitive material. Molecules 2018, $23,2064$. [CrossRef] [PubMed]

26. Buie, T.; McCune, J.; Cosgriff-Hernandez, E. Gelatin matrices for growth factor sequestration. Trends Biotechnol. 2020, 38 , 546-557. [CrossRef] 
27. Aldana, A.A.; Abraham, G.A. Current advances in electrospun gelatin-based scaffolds for tissue engineering applications. Int. J. Pharm. 2017, 523, 441-453. [CrossRef]

28. Noshadi, I.; Hong, S.; Sullivan, K.E.; Shirzaei Sani, E.; Portillo-Lara, R.; Tamayol, A.; Shin, S.R.; Gao, A.E.; Stoppel, W.L.; Black, L.D., III; et al. In vitro and in vivo analysis of visible light crosslinkable gelatin methacryloyl (GelMA) hydrogels. Biomater. Sci. 2017, 5, 2093-2105. [CrossRef]

29. Rehman, S.R.U.; Augustine, R.; Zahid, A.A.; Ahmed, R.; Tariq, M.; Hasan, A. Reduced graphene oxide incorporated GelMA hydrogel promotes angiogenesis for wound healing applications. Int. J. Nanomed. 2019, 14, 9603-9617. [CrossRef]

30. Dursun Usal, T.; Yucel, D.; Hasirci, V. A novel GelMA-pHEMA hydrogel nerve guide for the treatment of peripheral nerve damages. Int. J. Biol. Macromol. 2019, 121, 699-706. [CrossRef] 